EPJ Web of Conferences 43, 05005 (2013)

DOI: $10.1051 /$ epjconf/20134305005

(C) Owned by the authors, published by EDP Sciences, 2013

\title{
Pulsations in white dwarfs: Selected topics
}

\author{
H. Saio ${ }^{a}$
}

Astronomical Institute, Faculty of Science, Tohoku University, Sendai, Japan

\begin{abstract}
This paper presents a very brief overview of the observed properties of g-mode pulsations in variable white dwarfs. We then discuss a few selected topics: Excitation mechanisms (kappa- and convection- mechanisms), and briefly the effect of a strong magnetic field ( $\sim 1 \mathrm{MG})$ on g-modes as recently found in a hot DQ (carbon-rich atmosphere) white dwarf. In the discussion of excitation mechanisms, a simple interpretation for the convection mechanism is given.
\end{abstract}

\section{INTRODUCTION}

A white dwarf pulsates during the cooling evolution when it enters into instability ranges appropriate for its envelope composition. The pulsations are nonradial low-degree g-modes with periods ranging from $\sim 100$ to $\sim 1000$ seconds. Since the first white dwarf variable, HL Tau 76, was discovered by Landolt [18], many (presently 200) white dwarf pulsators were found, and as our understanding on the pulsations deepens, many articles continue to be published. The research in the field is still very active, trying to resolve the remaining many problems. In addition, much attention has been payed recently to the asteroseismological aspect of g-mode pulsations of white dwarfs, which gives us important information on the mass, rotation rate, cooling rate, etc. Two recent review papers, [9] and [31], thoroughly discuss the development of our asteroseismic understanding of these stars. Since giving a thorough review on the rich research field is beyond the author's ability, this paper picks out a few interesting topics after giving a brief overview on the properties of pulsations in white dwarfs.

\section{OVERVIEW ON THE PROPERTIES OF PULSATIONS IN WHITE DWARFS}

Pulsating white dwarfs are classified into four groups; from hotter to cooler: GW Vir stars, variable DB (helium-envelope) white dwarfs (DBVs), variable hot DQ (with carbon-rich atmosphere) white dwarfs (hot DQVs), and variable DA (hydrogen-envelope) white dwarfs (DAVs or ZZ Ceti stars). Figure 1 shows approximate positions of these groups on the Hertzsprung-Russell (HR) diagram together with a $0.57 \mathrm{M}_{\odot}$ evolutionary track. The hottest group, the GW Vir stars (sometimes called variable PG 1159 stars or DOVs) are variables among young hot white dwarfs having peculiar hydrogen-deficient (C/Orich) surface compositions (see [29] for a review). Among the four groups, the hot DQV is the newest one; the light variation of the prototype, SDSS J142625.7+1575218.3 was discovered in 2008 [19]. So far only four members are known in this group [8]. Figure 1 shows that the white dwarf pulsators lie, more or less, along the evolutionary track of $\sim 0.6 \mathrm{M}_{\odot}$, indicating the typical mass of the white dwarf pulsators are the same as that of the whole white dwarf population (e.g., [26]). We note, however, that recently, a very low mass $\left(\sim 0.17 \mathrm{M}_{\odot}\right)$ variable DA white dwarf was found [15].

\footnotetext{
a e-mail: saio@astr.tohoku.ac.jp
}

This is an Open Access article distributed under the terms of the Creative Commons Attribution License 2.0, which permits unrestricted use, distribution, and reproduction in any medium, provided the original work is properly cited. 


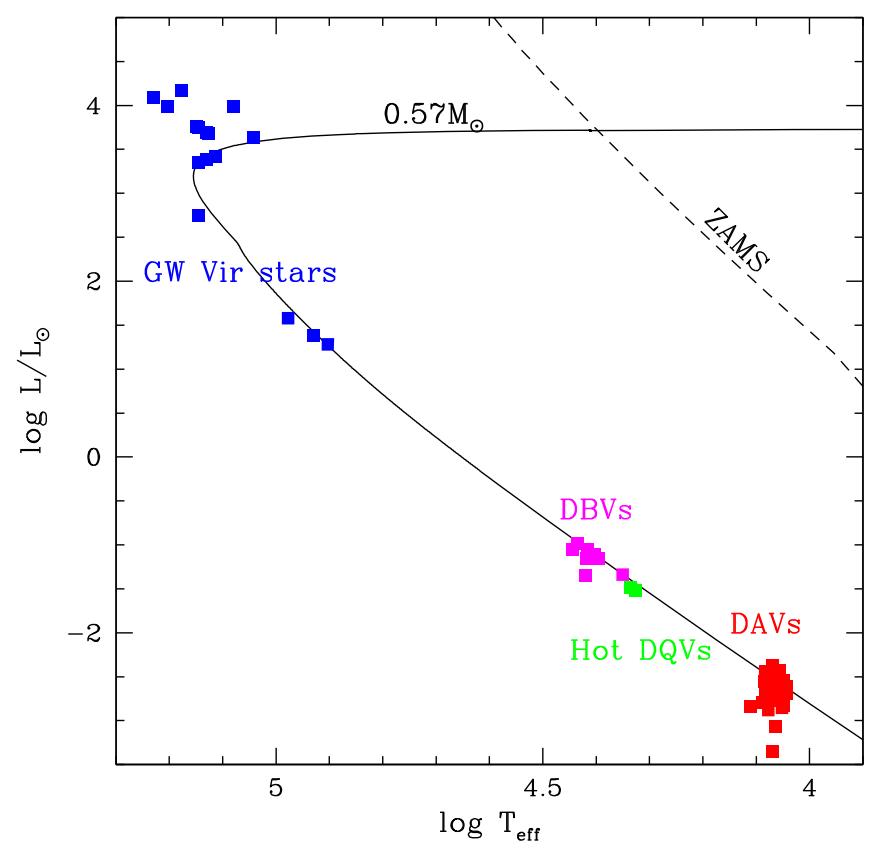

Figure 1. Approximate positions on the HR diagram of various types of pulsating white dwarfs with an evolutionary track of $0.57 \mathrm{M}_{\odot}$. The luminosity of each star was calculated from the effective temperature and surface gravity (assuming a mass of $0.6 \mathrm{M}_{\odot}$ for all stars) listed in [9] for GW Vir stars, DBVs, and DAVs, and in [6] for hot DQVs. The evolutionary track was obtained with the MESA code ([20]).

Figure 2 shows observed period ranges for various groups of white dwarf variables as a function of the effective temperature. Generally, a white dwarf pulsator shows multiple periods in a wide period range. The period ranges for different groups are: $\sim 300-5000$ s for GW Vir stars, $\sim 200-1000$ s for DBVs, and 100 - 1000 for DAVs. Among GW Vir variables, some stars are still contracting toward the 'knee'; they have longer periods because of their larger radii.

Also shown in Fig. 2 are the periods of $\mathrm{g}_{2}$ and $\mathrm{g}_{20}$ modes of $l=1$ in $0.6 M_{\odot}$ cooling models for reference. Except for contracting GW Vir stars, pulsations in white dwarfs are low to intermediate order g-modes with low latitudinal degree $l$. In the contracting GW Vir stars, the Brunt-Väisälä frequency in the core is still high so that g-modes in the observed period ranges of these stars have many nodes in the interior.

\subsection{Hot DQVs}

The light variations of the hot DQVs are somewhat peculiar (e.g., [19], [8]): each star shows only one or two periods, and the shorter period is close to half of the longer period, although those periods are within the period ranges of DBVs and DAVs. It was argued that the light variations could be explained either by pulsations or interacting binaries ([19], [1]). More recently, Green et al. [13] found a third frequency in the prototype hot DQV SDSS J142625.71+575218.3, which supports the pulsation hypothesis. The pulsation explanation is also supported by the nonadiabatic analysis of [10]; they found that g-modes with periods similar to observed ones are indeed excited in hot DQ white dwarf models. These recent findings indicate that at least some of the hot DQVs (including the prototype star) are very likely g-mode pulsators. Theoretically it is very interesting that the prototype star has a strong magnetic field ([7]). We will discuss briefly in $\S 4$ the interaction between g-mode pulsations and a magnetic field. 
Ageing Low Mass Stars: From Red Giants to White Dwarfs

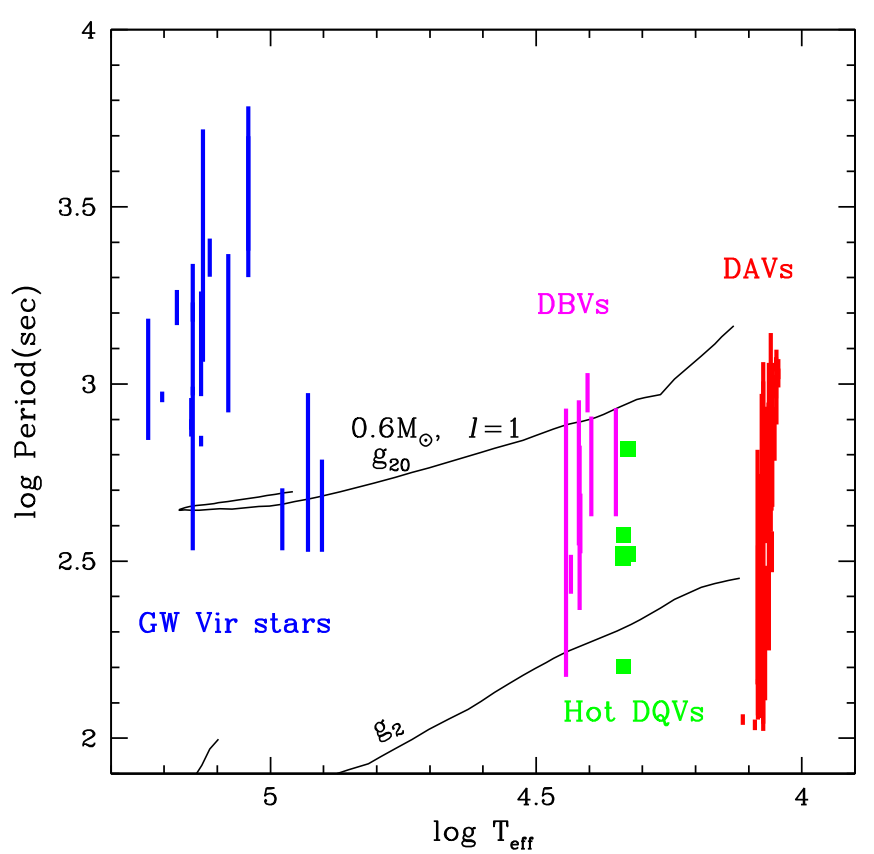

Figure 2. Period ranges observed in various types of white dwarf pulsators, plotted versus effective temperature. Also shown are the periods of dipole $\mathrm{g}_{2}$ and $\mathrm{g}_{20}$ modes of $0.6 M_{\odot}$ white dwarf cooling models. Effective temperatures and period ranges of the observed variables white dwarfs are adopted from [9].

\subsection{Period changes}

During its contraction, the period of a g-mode of a GW Vir star decreases. After passing the maximum effective temperature ('knee'), however, the period increases as the star cools because the Brunt-Väisälä frequency decreases. Because the evolution of a hot GW Vir star is rapid, the period change is detectable; Costa \& Kepler [4] measured directly a period-increase rate of $\sim 4 \mathrm{msec} / \mathrm{yr}$ for the $516 \mathrm{~s}$ main pulsation of GW Vir itself. A very rough estimate of the WD cooling rate yields

$$
d \ln T_{\mathrm{c}} / d t \propto L^{5 / 7}
$$

(e.g., [14]). This relation predicts the period-increase rate of a DAV should be slower than that of GW Vir by a factor of $\sim 10^{4}$, because the luminosity ratio is roughly $10^{-5}$. The prediction is roughly consistent with the observed increase rate of $\sim 0.1 \mu \mathrm{sec} / \mathrm{yr}$ as obtained for the DAV G117-B15A ([3]).

\subsection{Rotation rates}

One of the most important information derived from white dwarf oscillations is their rotation rates. The rotation frequency is obtained by measuring splittings of pulsation frequencies proportional to the azimuthal order $m$ (assuming rotation frequency is much smaller than the pulsation frequency). Since the first clear measurement for GW Vir ([30]) by the Whole Earth Telescope (WET) campaign observations, rotation frequencies have been obtained for several white dwarfs of various types (see references in [9]). In all of the cases, the rotation periods are much longer than the pulsation periods (the ratios are $\sim 200$ or larger), which justifies the linear analysis with respect to the rotation frequency. The corresponding total angular momentum ranges from $\sim 3 \times 10^{-3}$ to $\sim 0.02$ of the solar value, indicating these white dwarfs lost most angular momentum during the evolution before becoming white dwarfs. 


\section{EPJ Web of Conferences}

\section{EXCITATION MECHANISMS}

Excitation mechanisms of stellar pulsations are classified into the thermal processes (self-excitations) and the dynamical (forcing) processes. Dynamical processes include excitation by periodic tidal forces from a companion in a close binary system, and stochastic excitation by turbulent convection.

\subsection{Stochastic excitation for g-modes in white dwarfs?}

It is well known that the stochastic excitation is responsible for solar-like oscillations recently detected in many solar-like stars and red giants. The stochastic excitation is most efficient for pulsations with periods comparable to the characteristic timescale of convection in (energetic) outer layers. The frequency of oscillation most optimally excited by stochastic excitation, $v_{\max }$, can be scaled as

$$
v_{\max } \approx\left(\frac{R}{R_{\odot}}\right)^{-2} \frac{M}{M_{\odot}}\left(\frac{T_{\text {eff }}}{T_{\text {eff } \odot}}\right)^{-0.5} \times 3050 \mu \mathrm{Hz}
$$

(Kjeldsen \& Bedding [17]). Upon substituting typical parameters for ZZ Ceti stars (DAVs); $R \approx$ $0.01 R_{\odot}, M \approx 0.6 M_{\odot}$, and $T_{\text {eff }} \approx 12000 \mathrm{~K}$ into the above equation, we obtain $1 / v_{\max } \approx 0.08 \mathrm{sec}$. This is comparable with the convective turnover timescale of ZZ Ceti models [28]. However, $1 / \nu_{\max }$ is very much shorter than the observed period ranges of ZZ Ceti stars and of the other WD pulsators (see Fig. 2). This clearly indicates that stochastic excitation is ineffective for g-modes in white dwarfs.

\subsection{Excitation mechanisms by thermal processes}

During stellar pulsations, thermal energy is perturbed (exchanged), which leads to either self-excitation or damping of a perturbation. The most important equation for the process is the conservation of thermal energy

$$
T \frac{d S}{d t}=\epsilon-\frac{1}{\rho} \nabla \cdot \boldsymbol{F},
$$

where $T$ is temperature, $S$ entropy per unit mass, $\epsilon$ nuclear energy generation rate per unit mass, and $\boldsymbol{F}$ energy flux. Assuming that amplitudes of pulsations are small and the temporal variation is expressed as $\exp (i \sigma t)$, with angular frequency of pulsation $\sigma$, equation (3) may be linearized to

$$
i \sigma \frac{T M_{\mathrm{p}} C_{p}}{L} \frac{\delta S}{C_{p}} \approx \frac{M_{\mathrm{p}}}{L} \delta \epsilon-\frac{d}{d q}\left(\frac{\delta L_{r}}{L}\right),
$$

where $C_{p}$ is the specific heat per unit mass, $L_{r}$ local luminosity, $L$ luminosity at the surface, $M_{\mathrm{p}}$ the mass effectively involved in the pulsation, $q \equiv M_{r} / M_{\mathrm{p}}$, and $\delta$ means the Lagrangian perturbation of a quantity. In equation (4) we have neglected the horizontal energy flux perturbation, $\rho^{-1} \nabla_{h} \cdot \delta \boldsymbol{F}_{h}$ to simplify our qualitative discussion here.

The quantity $T M_{\mathrm{p}} C_{p} / L$ is the thermal timescale $\tau_{\text {th }}$ of the pulsating layer. The coefficient of $\delta S / C_{p}$ on the left hand side of equation (4) is proportional to the ratio of the thermal to dynamical timescale, $\tau_{\text {th }} / \Pi$, where $\Pi$ is the pulsation period. For most pulsators (except for very luminous ones) $\tau_{\text {th }} / \Pi \gg 1$ so that entropy perturbation $\left|\delta S / C_{p}\right|$ is generally very small (except for very near to the surface); in such cases, we can use adiabatic relations in evaluating $\delta \epsilon$ and $\delta L$; the approximation is called quasiadiabatic approximation.

As the pulsation amplitude is assumed to be proportional to $\exp (i \sigma t)$, the growth or decay of the pulsation amplitude is determined by the sign of the imaginary part $\sigma_{i}$ of the complex frequency $\sigma$; i.e., $\sigma_{\mathrm{i}}<0$ corresponds to excitation (or amplitude growth). Starting with equation (4) (or a similar one), 


\section{Ageing Low Mass Stars: From Red Giants to White Dwarfs}

we can express the imaginary part $\sigma_{\mathrm{i}}$ as

$$
\sigma_{\mathrm{i}}=-\frac{1}{2 \sigma_{\mathrm{r}}^{2} I} \int_{0}^{M} \frac{\delta T^{*}}{T}\left[\delta \epsilon-\frac{d \delta L_{r}}{d M_{r}}\right] d M_{r}
$$

where $\sigma_{\mathrm{r}}$ is the real part of frequency $\sigma$, and $I=\int_{0}^{M}|\xi|^{2} d M_{r}$ with $\xi$ being Lagrangian displacement vector (see e.g., [27] for details).

Since g-mode pulsations in white dwarfs, the condition $\tau_{\text {th }} / \Pi \gg 1$ is well satisfied, we use the quasi-adiabatic approximation; i.e., we use the adiabatic relations

$$
\frac{\delta \rho}{\rho}=\frac{1}{\Gamma_{1}} \frac{\delta P}{P}=\frac{1}{\Gamma_{3}-1} \frac{\delta T}{T},
$$

on the right hand side of equation (5), with $\Gamma_{1}=(\partial \ln P / \partial \ln \rho)_{S}$ and $\Gamma_{3}-1=\Gamma_{1} \nabla_{\mathrm{ad}}$, with adiabatic temperature gradient $\nabla_{\mathrm{ad}}=(\partial \ln T / \partial \ln P)_{S}$.

\subsubsection{Epsilon mechanism}

In the quasi-adiabatic approximation, the first term of the right hand side of equation (5) is written as

$$
\frac{\delta T^{*}}{T} \delta \epsilon=\frac{\delta T^{*}}{T}\left(\epsilon_{T} \frac{\delta T}{T}+\epsilon_{\rho} \frac{\delta \rho}{\rho}\right)=\epsilon\left(\epsilon_{T}+\frac{\epsilon_{\rho}}{\Gamma_{3}-1}\right)\left|\frac{\delta T}{T}\right|^{2}>0,
$$

where $\epsilon_{T} \equiv(\partial \ln \epsilon / \partial \ln T)_{\rho}(>0)$ and $\epsilon_{\rho} \equiv(\partial \ln \epsilon / \partial \ln \rho)_{T}(>0)$. Obviously, this term, is always positive; i.e., nuclear energy generation always drives oscillations; this is called the epsilon mechanism. Although the mechanism works wherever nuclear reactions are active, the pulsation amplitude tends to be small in such high temperature layers so that the epsilon mechanism is very weak for most of the global pulsations. Nuclear shell burning in some pre-white dwarfs (including contracting GW Vir stars) excites low-order g-modes [16]; no observational evidence of such short-period oscillations could be gathered as of now.

\subsubsection{Radiative effects; kappa-mechanism}

The second term on the right hand side of equation (5) is important in the stellar envelope. The term indicates that if the outward energy flow is blocked (i.e., $d \delta L / d M_{r}<0$ ) in the phase of $\delta T>0$ (i.e., in the compressed phase), the associated layers drive the pulsation. The thermal energy in the stellar envelope is carried by radiation and convection so that we can write

$$
L_{r}=L_{\mathrm{r} ; \mathrm{rad}}+L_{\mathrm{r} ; \mathrm{conv}}, \quad \delta L_{r}=\delta L_{\mathrm{r} ; \mathrm{rad}}+\delta L_{\mathrm{r} ; \mathrm{conv}} .
$$

In the following we discuss the radiative and the convective part separately.

Firstly we discuss radiative effects in the envelope where no nuclear reaction occurs and energy is carried by radiation only; then $L_{r}=L_{r ; \text { rad }}=L$ (constant). Assuming diffusive radiative transfer, we express the radiative luminosity as

$$
L_{r ; \mathrm{rad}}=-16 \pi^{2} r^{2} \frac{a c}{3 \kappa} \frac{d T^{4}}{d M_{r}} .
$$

Linearizing equation (9) yields in the quasi-adiabatic limit

$$
\frac{\delta L_{r ; \mathrm{rad}}}{L} \approx\left(4-\kappa_{T}-\frac{\kappa_{\rho}}{\Gamma_{3}-1}\right) \frac{\delta T}{T}+4 \frac{\xi_{r}}{r}+\frac{d(\delta T / T) / d \ln M_{r}}{d \ln T / d \ln M_{r}},
$$

where $\kappa_{T}=(\partial \ln \kappa / \partial \ln T)_{\rho}$ and $\kappa_{\rho}=(\partial \ln \kappa / \partial \ln \rho)_{T}$. 
Using equation (10) in the radiative part of equation (5), we obtain

$$
\begin{aligned}
\left.-\sigma_{\mathrm{i}} \text { (radiation) }\right) & \approx-\frac{L}{2 \sigma_{\mathrm{r}}^{2} I} \int_{0}^{M} \frac{\delta T^{*}}{T} \frac{d}{d M_{r}}\left[\left(4-\kappa_{T}-\frac{\kappa_{\rho}}{\Gamma_{3}-1}\right) \frac{\delta T}{T}+4 \frac{\xi_{r}}{r}+\frac{d(\delta T / T) / d \ln M_{r}}{d \ln T / d \ln M_{r}}\right] d M_{r} \\
& \approx \frac{L}{2 \sigma_{\mathrm{r}}^{2} I} \int_{0}^{M}\left|\frac{\delta T}{T}\right|^{2} \frac{d}{d M_{r}}\left(\kappa_{T}+\frac{\kappa_{\rho}}{\Gamma_{3}-1}\right) d M_{r} .
\end{aligned}
$$

For the last equality, only leading terms are kept. This equation indicates that in a weak nonadiabatic environment, radiative energy flow drives pulsations mainly when

$$
\frac{d}{d r}\left(\kappa_{T}+\frac{\kappa_{\rho}}{\Gamma_{3}-1}\right)>0
$$

Since this driving occurs due to changes in opacity, this is called $\kappa$-mechanism or opacity mechanism. The g-mode pulsations of GW Vir stars, where convection is inefficient, are exited by the $\kappa$-mechanism at C/O-opacity bump at $\sim 2 \times 10^{6} \mathrm{~K}$ (e.g., [22], [11] ). The $\kappa$-mechanism in GW Vir stars is strong enough to excite g-modes only if the $\mathrm{C} / \mathrm{O}$ abundance in the driving layers is high enough, so that pulsators and non-pulsators co-exist in the same region on the HR diagram depending on the initial composition and the strength of elemental diffusion (e.g., [21]).

\subsubsection{Convection mechanism in white dwarfs}

Efficient convection zones are present in the envelopes of DA and DB pulsators, for them $L_{\mathrm{r} \text {;conv }} \gg$ $L_{\mathrm{r} \text {;rad }}$. Therefore, the perturbation of the convective luminosity, $\delta L_{\mathrm{r} \text {; conv }}$, is important in equation (5), and hence plays an important role for the stability of g-modes in DA and DB variables. Evaluating pulsational perturbations of convective luminosity $\delta L_{\mathrm{r}, \mathrm{conv}}$ in general is very complicated and uncertain due to our poor understanding of turbulent convection in stars. Fortunately, convection zone in white dwarfs has a 'good' property; i.e., the convective turnover time is much shorter than the periods of the low-degree g-modes. This means that the convective flux is instantaneously adjusted during the pulsation. Utilizing this property, Brickhill [2] and Goldreich \& Wu [12] found the 'convection mechanism' for the excitation of g-modes in DA variables. We discuss here the convection mechanism from a slightly different point of view.

A simple form of the convective flux based on the mixing-length theory may be written as

$$
F_{\mathrm{conv}} \approx C_{p} \rho \overline{v_{\mathrm{conv}} \Delta T} \approx \frac{\alpha^{2}}{4 \sqrt{2}} C_{p}^{3 / 2} \nabla_{\mathrm{ad}}^{1 / 2} \rho T^{3 / 2}\left(\nabla_{T}-\nabla_{\mathrm{ad}}\right)^{3 / 2}
$$

where $v_{\text {conv }}$ is the characteristic velocity of convective eddies, $\Delta T$ is the temperature excess of an eddy, $\nabla_{T} \equiv d \ln T / d \ln P, \nabla_{\mathrm{ad}}=\left(\Gamma_{3}-1\right) / \Gamma_{1}$, adiabatic temperature gradient, and $\alpha$ the ratio of mixinglength to pressure scale height. Using the relation between the super-adiabatic temperature gradient $\left(\nabla_{T}-\nabla_{\mathrm{ad}}\right)$ and entropy gradient;

$$
\frac{d S}{d r}=-\frac{C_{p} g \rho}{P}\left(\nabla_{T}-\nabla_{\mathrm{ad}}\right)
$$

the convective flux can be written as

$$
F_{\text {conv }} \approx 2^{-5 / 2} \alpha^{2} g^{-3 / 2} \nabla_{\text {ad }}^{1 / 2}(P T)^{3 / 2} \rho^{-1 / 2}\left(-\frac{d S}{d r}\right)^{3 / 2}
$$

where $g=G M_{r} / r^{2}$ is the local gravitational acceleration. Assuming $d S / d r$ and $\alpha$ are constant during $\mathrm{g}$-mode pulsations, we obtain the adiabatic perturbation of the convective flux as

$$
\frac{\delta F_{\text {conv }}}{F_{\text {conv }}}=\frac{1}{2}\left[3\left(1+\nabla_{\mathrm{ad}}\right)-\frac{1}{\Gamma_{1}}+\left(\frac{\partial \ln \nabla_{\mathrm{ad}}}{\partial \ln P}\right)_{T}+\nabla_{\mathrm{ad}}\left(\frac{\partial \ln \nabla_{\mathrm{ad}}}{\partial \ln T}\right)\right] \frac{\delta P}{P} .
$$




\section{Ageing Low Mass Stars: From Red Giants to White Dwarfs}

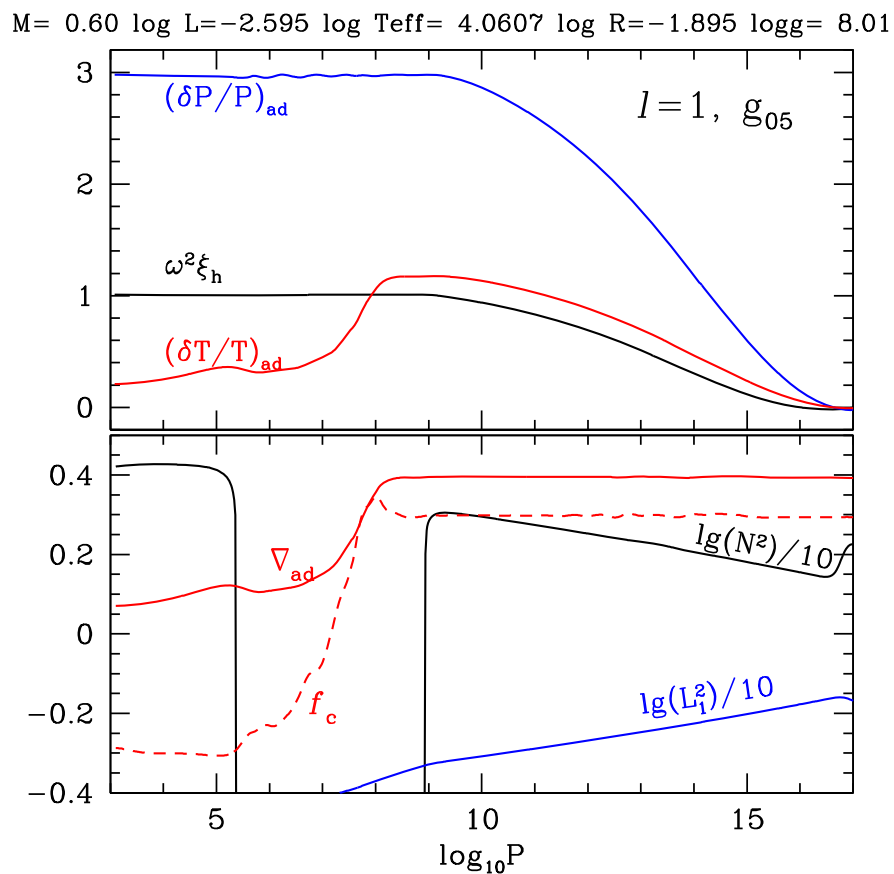

Figure 3. Top panel: spatial runs of pressure and temperature perturbations and horizontal displacement for the adiabatic dipole $\mathrm{g}_{5}$ mode in a DA white dwarf model of $0.6 M_{\odot}$ (Fontaine 2012; private communication). The bottom panel shows $f_{\mathrm{c}}$ given in equation (18) with Brunt-Väisälä and Lamb frequencies $(\ell=1)$. The model has a convection zone $(5.35 \lesssim \log P \lesssim 9)$ due to hydrogen ionization.

Since the convection zone in a white dwarf is geometrically thin, we can neglect the effect of sphericity; i.e., $\delta L_{\mathrm{r} \text {;conv }} / L=\delta F_{\text {conv }} / F_{\text {conv }}$. Substituting these relations into equation (5), we obtain the effect of convective energy transport on the stability as

$$
-\sigma_{\mathrm{i}}(\text { convection }) \approx-\frac{L}{2 \sigma_{\mathrm{r}}^{2} I} \int_{\mathrm{CZ}} \frac{\delta T^{*}}{T} \frac{d}{d M_{r}}\left(\frac{\delta F_{\text {conv }}}{F_{\text {conv }}}\right) \approx-\frac{L}{2 \sigma_{\mathrm{r}}^{2} I} \int_{\mathrm{CZ}} \nabla_{\mathrm{ad}}\left(\frac{\delta P}{P}\right)^{2} \frac{d f_{\mathrm{c}}}{d M_{r}},
$$

where

$$
f_{\mathrm{c}} \equiv \frac{3}{2} \nabla_{\mathrm{ad}}-\frac{1}{2 \Gamma_{1}}+\frac{1}{2}\left(\frac{\partial \ln \nabla_{\mathrm{ad}}}{\partial \ln P}\right)_{T}+\frac{1}{2} \nabla_{\mathrm{ad}}\left(\frac{\partial \ln \nabla_{\mathrm{ad}}}{\partial \ln T}\right)_{P},
$$

$\int_{\mathrm{CZ}}$ means integration through the convection zone. The second relation in equation (17) is obtained from the fact that $\delta P / P$ is nearly constant in the convection zone as seen in Fig. 3 (as first found by [2]). The quantity $f_{\mathrm{c}}\left[=\left(\delta F_{\text {conv }} / F_{\text {conv }}\right) /(\delta P / P)-3 / 2\right]$ decrease outward in the convection zone except around a small peak at $\log P \approx 8$ produced by a positive value of the gradient $\left(\partial \ln \nabla_{\mathrm{ad}} / \partial \ln T\right)_{P}$. The outward decrease of $f_{\mathrm{c}}$ mainly comes from a decrease in $\nabla_{\text {ad }}$ due to the H-ionization. This property makes the right hand side of equation (17) always positive; in other word the convective flux perturbation drives pulsations in the white dwarf as long as the pulsation period is much longer than the convection turnover time. This is the convection mechanism for g-mode pulsations in DA variables, which was found by Brickhill [2] and further discussed by Goldreich \& Wu [12]. The cause of the convective driving can be considered as the effect of partial ionization of hydrogen which absorbs energy in the compressed phase and release it in the expanded phase.

In order for g-modes to be excited by convective driving, it must exceed radiative damping that occurs below the convection zone. The optimal condition for the convection (and $\kappa$ ) mechanism is 
that thermal timescale is comparable to the pulsation period in the driving zone (see [5] for detailed discussions). If the effective temperature is too high, the oscillation periods are comparable to the thermal timescale in the radiative damping zone below the convection zone and hence radiative damping exceeds convective driving. Therefore, the blue edge (high-temperature boundary of the instability strip in the HR diagram) occurs at a model for which periods of low-order g-modes are equal to a few times of the thermal timescale at the bottom of the convection zone ([12]). The predicted blue edge is consistent with the observed one $\sim 12000 \mathrm{~K}$ of the ZZ Ceti instability strip. However, the mechanism predicts a red edge to be much cooler than the observed one; theory predicts the red edge at $\sim 6000 \mathrm{~K}$ while observed one is $\sim 11000 \mathrm{~K}[28]$. The discrepancy has not been resolved yet, but it may be attributed to mechanical damping by Reynolds stress.

We note that the stability of g-modes is not different even if the frozen convection approximation is used ([28]), in which convective flux perturbation is neglected (opposite extreme to the instantaneous adjustment approximation) so that only the $\kappa$-mechanism with radiative luminosity ( $\left.\ll L_{\text {conv }}\right)$ works. This is understandable, because both mechanism works at the same layers (i.e., partial ionization zone), due to a change in the opacity derivatives in the $\kappa$-mechanism, while the decrease of the adiabatic temperature gradient is pertinent for the convection mechanism.

Before moving to the next topic, it should be mentioned that a new excitation mechanism is proposed by Shibahashi ([25] and these proceedings) related to double diffusive (overstable) convection; the mechanism seems to be effective in white dwarfs in a effective temperature range of 45,000-30,000K.

\section{THE EFFECT OF MAGNETIC FIELDS}

Dufour et al. [7] found a strong magnetic field of $\approx 1.2 \mathrm{MG}$ in the prototype hot DQ variable SDSS $\mathrm{J} 142625.71+575218.3$ which shows light variations with periods of $418 \mathrm{~s}$, 320s and 209s (the last one is the harmonic of the first one, [13]). In the presence of a magnetic field $\boldsymbol{B}$, the Lorentz force term enters into the equation of motion;

$$
\frac{d^{2} \xi}{d t^{2}}=-\left(\frac{1}{\rho} \nabla p\right)^{\prime}+\frac{1}{4 \pi \rho}\left(\nabla \times \boldsymbol{B}^{\prime}\right) \times \boldsymbol{B}_{0} \quad \text { with } \quad \frac{\partial \boldsymbol{B}^{\prime}}{\partial t}=\nabla \times\left(\frac{d \xi}{d t} \times \boldsymbol{B}_{0}\right),
$$

where the prime' indicates the Eulerian perturbation and $\xi$ the displacement. The Lorentz force affects nonradial pulsations and introduces additional waves.

Although the effects of strong magnetic fields on high-order p-modes in rapidly oscillating Ap (roAp) stars have been investigated (e.g., [24] for a review), coupling with g-modes in white dwarfs have never been studied before. Figure 4 compares envelope properties of a magnetized hot DQ model with those of a typical roAp model. A polar field strength of $B_{\mathrm{p}}=1.2 \mathrm{MG}$ is assumed for the white dwarf model, while $5 \mathrm{kG}$ is assumed for the roAp model. Because of the high density in the white dwarf, magnetically dominant layers (where $V_{\mathrm{A}}>C_{\mathrm{s}}$ with $V_{\mathrm{A}}$ and $C_{\mathrm{s}}$ being Alfvén and sound speed, respectively) extend up to the middle of the convection zone; i.e., above the boundary of the g-mode propagation cavity. On the other hand, the boundary enters well into the p-mode propagation cavity in the roAp star model. This seems to indicate that the magnetic effect on g-modes might not be very strong in white dwarfs even with a MG field.

As a first preliminary trial, a nonadiabatic analysis code used for axisymmetric pulsations of roAp stars ([23]) was applied to the hot DQ model. Figure 5 shows the eigenfunction of a nearly dipole $\mathrm{g}_{3}$ mode with $B_{\mathrm{p}}=1.1 \mathrm{MG}$. In the presence of a magnetic field, the angular dependence of a pulsation mode cannot be expressed by a single spherical harmonic so that the eigenfunction is represented by a superposition of terms associated various degrees of spherical harmonics. The small inset in the top panel of Fig. 5 shows surface amplitude $(\times)$ and kinetic energy $(\bullet)$ of each term of degree $l$, indicating that components associated with degree $l$ larger than unity contribute considerably to the mode so that the amplitude distribution is deformed considerably from the dipole character. 
Ageing Low Mass Stars: From Red Giants to White Dwarfs
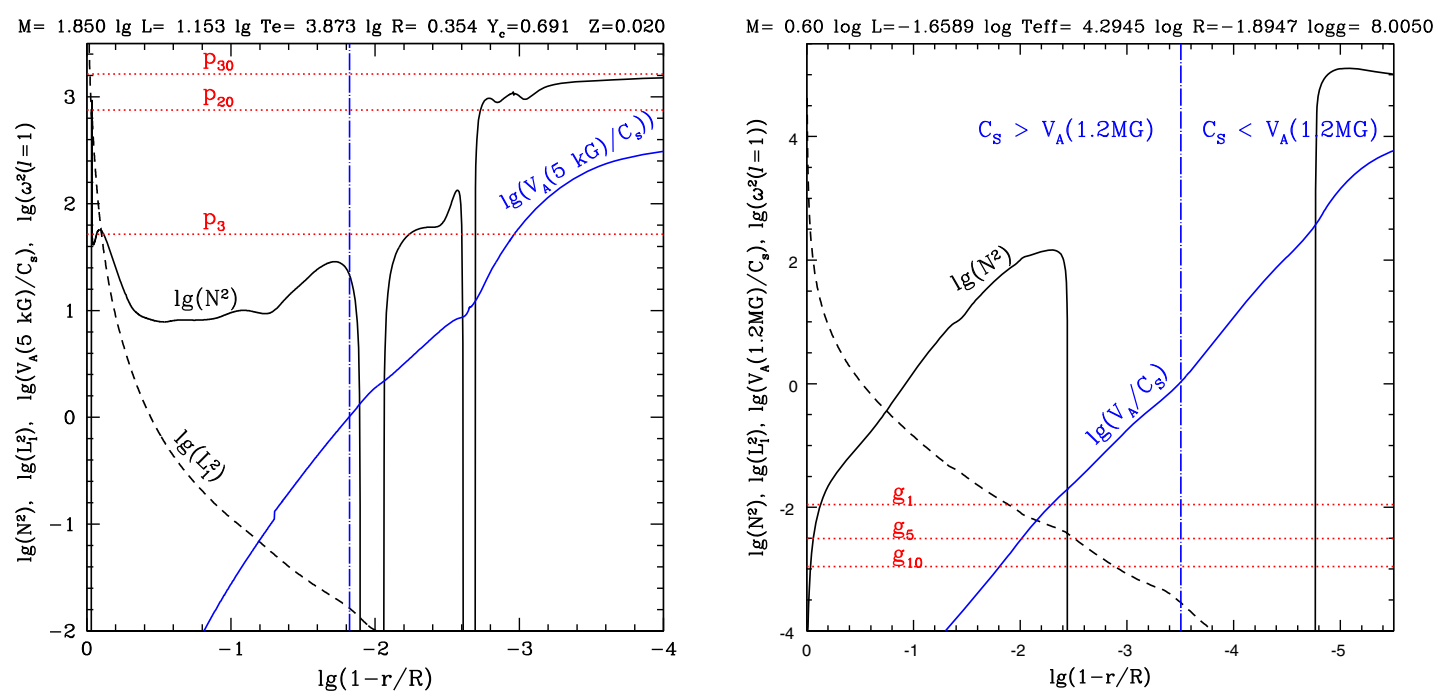

Figure 4. The propagation diagram of a model (Fontaine; private communication) for hot DQ white dwarf (right panel) is compared with that of a typical roAp star model (left panel). Polar strengths of magnetic fields of 1.2 MG for the hot DQ model and $5 \mathrm{kG}$ for the roAp star model were adopted. Vertical dashed lines divide between magnetic energy dominant upper part $\left(C_{\mathrm{s}}<V_{\mathrm{A}}\right)$ and thermal energy dominant lower part $\left(C_{\mathrm{s}}>V_{\mathrm{A}}\right)$, where $C_{\mathrm{s}}$ and $V_{\mathrm{A}}$ are sound and Alfvén speed, respectively.

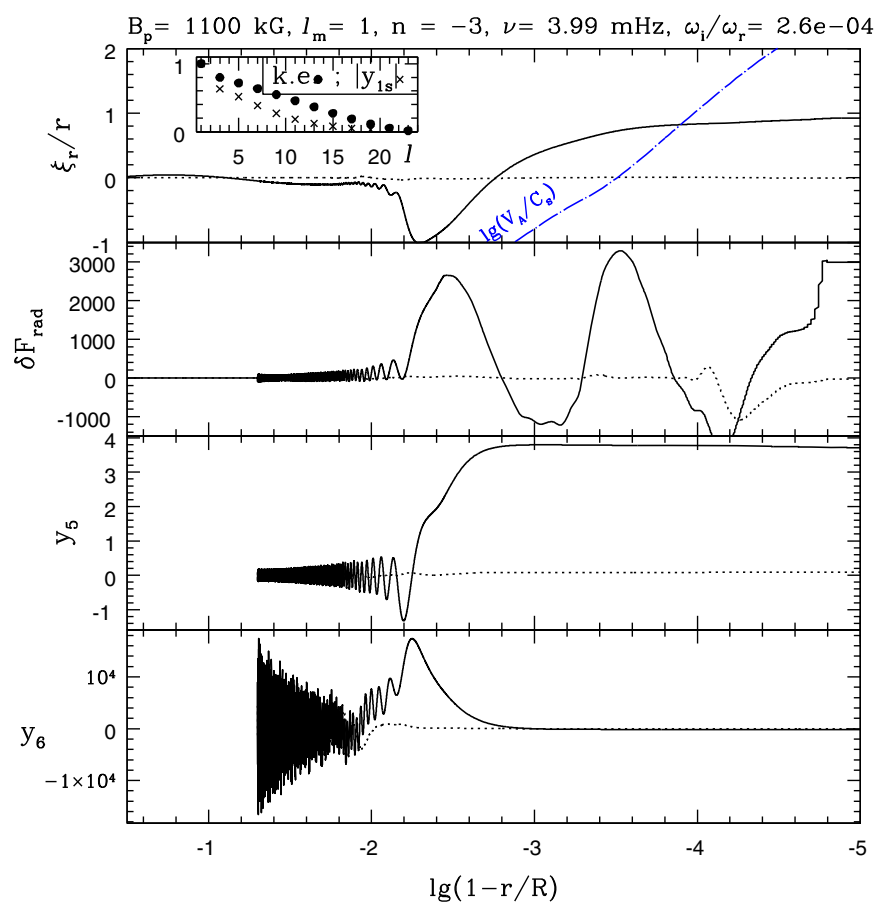

Figure 5. Eigenfunction components of the dipole $\mathrm{g}_{3}(\Pi=251 \mathrm{sec})$ mode in a hot DQ white dwarf model of $0.6 M_{\odot}$ with a polar magnetic field strength of 1.1 MG. Solid and dotted lines stand for real and imaginary parts, respectively. 
The variables $y_{5}$ and $y_{6}$ are related to perturbations of the magnetic field. They show very rapid spatial oscillations due to magnetic slow waves propagating inward. Assuming the slow waves to be dissipated before reaching the stellar center, running wave conditions are imposed at $r / R=0.95$ for $y_{5}$ and $y_{6}$. The amplitude of the slow waves at the boundary appears to be much larger than in the case of for roAp star models, indicating a strong coupling between the g-mode and the magnetic field. Hence, further detailed investigations are needed.

I am very grateful to Gilles Fontaine for providing me with some white dwarf models and for helpful comments, and to Alfred Gautschy for helpful comments.

\section{References}

[1] Barlow, B.N., Dunlap, B.H., Rosen, R. \& Clemens, J.C., ApJ, 688, (2008) L95

[2] Brickhill, A.J., MNRAS, 204, (1983) 537

[3] Córsico, A.H., Althaus, L.G., Miller Bertolami, M.M., et al., MNRAS, 424, (2012) 2792

[4] Costa, J.E.S. \& Kepler, S. O., A\&A, 489, (2008) 1225

[5] Cox, J.P., Rep. Prog. Phys., 37, 563

[6] Dufour, P., Fontaine, G., Liebert, J., Schmidt, G.D. \& Behara, N., ApJ, 683, (2008) 978

[7] Dufour, P., Fontaine, G., Liebert, J., Williams, K. \& Lai, D.K., ApJ, 683, (2008) L167

[8] Dufour, P., Béland, S., Fontaine, G., Chayer, P., Bergeron, P., ApJ, 733, (2011) L19

[9] Fontaine, G. \& Brassard, P., PASP, 120, (2008) 1043

[10] Fontaine, G. \& Brassard, P. \& Dufour, P., A\&A, 483, (2008) L1

[11] Gautschy, A., A\&A, 320, (1997) 811

[12] Goldreich, P. \& Wu, Y., ApJ, 511, (1999) 904

[13] Green, E.M., Dufour, P., Fontaine, G. \& Brassard, P., ApJ, 702, (2009) 1593

[14] Hansen, C.J., Kawaler, S.D. \& Trimble, V. Stellar Interiors; Physical Principles, Structure, and Evolution (Springer-Verlag, New York 2004)

[15] Hermes, J.J., Montgomery, M.H., Winget, D.E., Brown, W.R., et al. ApJ, 750, (2012) L28

[16] Kawaler, S.D., ApJ, 334, (1988) 220

[17] Kjeldsen, H. \& Bedding, T. R., A\&A, 293, (1995) 87

[18] Landolt, A.U. ApJ, 153, (1968) 151

[19] Montgomery, M.H., Williams, K.A., Winget, D.E., et al., ApJ, 678, (2008) L51

[20] Paxton, B., Bildsten, L., Dotter, A., Herwig, F., Lesaffre, P. \& Timmes, F., ApJS, 192, (2011) 3

[21] Quirion, P.-Q., Fontaine, G. \& Brassard, P. ApJ, 755, (2012) 128

[22] Saio, H., in ASP Conf. Ser. 96, (ASP, San Francisco1996) 361

[23] Saio, H., MNRAS, 360, (2005) 1022

[24] Saio, H., JPCS, 118, (2008) 12018

[25] Shibahashi, H., AIP Conf. Proc., 948, (2007) 35

[26] Tremblay, P.-E., Bergeron, P. \& Gianninas, A., ApJ, 730, (2011) 128

[27] Unno, W., Osaki, Y., Ando, H., Saio, H., \& Shibahashi, H., Nonradial Pulsations of Stars (University of Tokyo Press, Tokyo 1989)

[28] Van Grootel, V., Dupret, M.-A., Fontaine, G., Brassard, P., et al., A\&A 539, (2012) A87

[29] Werner, K. \& Herwig, F., PASP 118, (2006) 183

[30] Winget, D.E., Nather, R.E., Clemens, J.C., et al., ApJ, 378, (1991) 326

[31] Winget, D.E. \& Kepler, S.O., ARA\&A 46, 157 